\title{
GCU
}

Glasgow Caledonian

University

University for the Common Good

\section{Why older adults spend time sedentary and break their sedentary behavior: a mixed methods approach using life-logging equipment}

Dontje, Manon L.; Leask, Calum F.; Harvey, Juliet; Skelton, Dawn A.; Chastin, Sebastien F. M.

Published in:

Journal of Aging and Physical Activity

DOI:

10.1123/japa.2016-0267

Publication date:

2018

Document Version

Author accepted manuscript

Link to publication in ResearchOnline

Citation for published version (Harvard):

Dontje, ML, Leask, CF, Harvey, J, Skelton, DA \& Chastin, SFM 2018, 'Why older adults spend time sedentary and break their sedentary behavior: a mixed methods approach using life-logging equipment', Journal of Aging and Physical Activity, vol. 26, no. 2, pp. 259-266. https://doi.org/10.1123/japa.2016-0267

\section{General rights}

Copyright and moral rights for the publications made accessible in the public portal are retained by the authors and/or other copyright owners and it is a condition of accessing publications that users recognise and abide by the legal requirements associated with these rights.

Take down policy

If you believe that this document breaches copyright please view our takedown policy at https://edshare.gcu.ac.uk/id/eprint/5179 for details

of how to contact us. 
"Why Older Adults Spend Time Sedentary and Break Their Sedentary Behavior: A Mixed Methods Approach Using LifeLogging Equipment" by Manon Dontje et al.

Note: This article will be published in a forthcoming issue of the Journal of Aging and Physical Activity. This article appears here in its accepted, peer-reviewed form, as it was provided by the submitting author. It has not been copy edited, proofed, or formatted by the publisher.

Section: Original Research

ArticleTitle: Why Older Adults Spend Time Sedentary and Break Their Sedentary Behavior: A Mixed Methods Approach Using Life-Logging Equipment

Authors: Manon L. Dontje ${ }^{1}$, Calum F. Leask ${ }^{1}$, Juliet Harvey ${ }^{1}$, Dawn A. Skelton ${ }^{1}$, Sebastien F.M. Chastin $^{1}$

Affiliations: ${ }^{1}$ School of Health and life Science, Institute of Applied Health Research, Glasgow Caledonian University, Glasgow, UK

Running Head: Sedentary behavior in older adults

Journal: Journal of Aging and Physical Activity

Acceptance Date: August 21, 2017

C2017 Human Kinetics, Inc.

DOI: http://dx.doi.org/10.1123/japa.2016-0267 
"Why Older Adults Spend Time Sedentary and Break Their Sedentary Behavior: A Mixed Methods Approach Using LifeLogging Equipment" by Manon Dontje et al.

Journal of Aging and Physical Activity

(c) 2017 Human Kinetics, Inc.

Sedentary behavior in older adults

Abstract

Older adults are recommended to reduce their sedentary time to promote healthy ageing. To develop effective interventions identifying when, why, and how older adults are able to change their sitting habits is important. The aim of this mixed-method study was to improve our understanding of reasons for (breaking) sedentary behavior in older adults. Thirty older adults $(74.0( \pm 5.3)$ years old, $73 \%$ women) were asked about their believed reasons for (breaking) sedentary behavior, and about their actual reasons when looking at a personal storyboard with objective records of activPAL monitor data and time-lapse camera pictures showing all their periods of sedentary time in a day. The most often mentioned believed reason for remaining sedentary was television/radio (mentioned by $48.3 \%$ ), while eating/drinking was most often mentioned as actual reason (96.6\%). Only $17.2 \%$ believed that food/tea preparation was a reason to break up sitting, while this was an actual reason for $82.8 \%$ of the study sample. Results of this study show that there is a discrepancy between believed and actual reasons for (breaking) sedentary behaviour. These findings suggest developing interventions utilizing the actual reasons for breaking sedentary behaviour to reduce sedentary time in older adults.

Keywords: Sedentary lifestyle, Aged, Behavior, Accelerometry, Interviews as topic 
"Why Older Adults Spend Time Sedentary and Break Their Sedentary Behavior: A Mixed Methods Approach Using LifeLogging Equipment" by Manon Dontje et al.

Journal of Aging and Physical Activity

(C) 2017 Human Kinetics, Inc.

Sedentary behavior in older adults

Why older adults spend time sedentary and break their sedentary behavior: a mixed methods approach using life-logging equipment.

Sedentary behavior is defined as any waking activity characterized by an energy expenditure $\leq 1.5$ metabolic equivalents and a sitting or reclining posture (Sedentary Behaviour Research, 2012). Older adults are the most sedentary segment of society. Older people ( $>65$ years) spend over $75 \%$ of their waking day sedentary (Davis et al., 2011; Harvey, Chastin, \& Skelton, 2015; Matthews et al., 2008). Current evidence suggests that for older adults, prolonged sedentary behavior is associated with less successful ageing, several chronic diseases and all-cause mortality, regardless of daily physical activity (Chau et al., 2013; de Rezende, Rey-López, Matsudo, \& Luiz, 2014; Dogra \& Stathokostas, 2012). Several national and international health guidelines explicitly recommend that older adults should reduce their sedentary time and break prolonged periods of sitting to promote healthy ageing and well-being (Australian Government, Department of Health, 2014; Department of Health, Physical Activity, Health Improvement and Protection, 2011; Department of Health, 2011).

In order to develop effective interventions, it is crucial to identify when, why, and how older adults are most likely to be able to change their sitting habits. To date, only two qualitative studies have investigated determinants of sitting and barriers and motivators to change sedentary behavior in older adults (Chastin, Fitzpatrick, Andrews, \& DiCroce, 2014; Greenwood-Hickman, Renz, \& Rosenberg, 2015). There appears to be a difference in determinants, barriers and motivators for sedentary behavior and for moderate to vigorous physical activity. This difference might be due to the ubiquitous nature of sedentary behavior, which occurs continuously during the day, compared to finite and set periods of moderate to vigorous physical activity incidental to daily living or exercise during the day or week. Also, physical activity requires at least some conscious effort to do, whereas sedentary behavior is often a state of rest and requires less thinking, preparing, planning, or effort. This suggests that interventions to reduce sedentary behavior need to be integrated into daily life. In addition, both studies suggested that not all periods of sitting are equally modifiable, due to personal, social, and environmental factors. Therefore, it is necessary to examine why older adults are 
"Why Older Adults Spend Time Sedentary and Break Their Sedentary Behavior: A Mixed Methods Approach Using LifeLogging Equipment" by Manon Dontje et al.

Journal of Aging and Physical Activity

(c) 2017 Human Kinetics, Inc.

Sedentary behavior in older adults

sedentary, and what they perceive as natural reasons to break up their sitting. These natural reasons could be used as starting points of interventions to increase the number of breaks without interfering too much with daily routine.

Obtaining this information accurately using solely qualitative research methods is challenging because older adults are often unaware of exactly how long, where, when and why they sit (Chastin et al., 2014; Greenwood-Hickman et al., 2015). Older adults find recollecting their sitting cognitively challenging (van Uffelen, Heesch, Hill, \& Brown, 2011). Comparisons between self-reported and objective measures of sedentary behavior have shown that older adults underestimate the time they spend sitting (Harvey et al., 2015). Objective measures combining accelerometers and time-lapse cameras are useful to quantify sedentary behavior in a specific context (Doherty et al., 2013; Leask, Harvey, Skelton, \& Chastin, 2015), but such life-logging equipment provides no information on perceived motivators and barriers. However, the data from such sensors have shown to be effective in helping adults recollect and reconstruct their day (Kelly et al., 2015), including older adults and individuals with dementia (Berry et al., 2007; Browne et al., 2011). Combining qualitative research methods with objective measurements of sedentary behavior, referred to as triangulation, micro-macro link, or mixed methods approach (Erzberger \& Prein, 1997), offers the opportunity to gain greater insight into sedentary behavior. One way of doing this is allowing older adults to reflect on their own objective records of their daily behavior.

The aim of this study was to improve our understanding of reasons for (breaking) sedentary behavior in older adults, by utilizing life-logging equipment (objective activity monitor and time-lapse camera) and a mixed-method approach. This in-depth information can be used to develop effective interventions to change sedentary behavior.

\section{Methods}

\section{Study sample}


"Why Older Adults Spend Time Sedentary and Break Their Sedentary Behavior: A Mixed Methods Approach Using LifeLogging Equipment" by Manon Dontje et al.

Journal of Aging and Physical Activity

(c) 2017 Human Kinetics, Inc.

Sedentary behavior in older adults

A convenience sample of older adults was recruited between March and April 2013 in senior clubs and bingo halls within the Glasgow area. Inclusion criteria were: (a) $\geq 65$ years of age, (b) independently living (community-dwelling), (c) Elderly Mobility Scale (EMS) score >14 (Smith, 1994), and (d) 6 Item Cognitive Impairment Test (6-CIT) score <8 (Brooke \& Bullock, 1999). Exclusion criteria were: (a) acute coronary syndrome/cardiovascular event within the last month, (b) neurological conditions, (c) allergies to adhesive tape (necessary for attaching the activity monitor), and (d) currently receiving a multidisciplinary rehabilitation program for any condition. This study complied with the principles outlined in the Declaration of Helsinki and was approved by the Glasgow Caledonian University School of Health and Life Sciences Ethics Committee. All participants gave their written informed consent. The protocol and data collection complied with the framework developed for health behavior research involving wearable cameras (Kelly et al., 2013).

\section{Study design}

This study used a mixed methods approach over a two day protocol. On day 1, the pattern of sedentary behavior was objectively measured using a combination of an activity monitor (activPAL physical activity monitor, PAL Technologies Ltd, Glasgow, Scotland) and a time-lapse camera (ViconRevue, Vicon Motion Systems Ltd, Oxford, United Kingdom) to develop an accurate visual depiction and frequencies of the participants' pattern of sitting on that day. On day 2, a 1- hour semistructured interview took place to assess reasons (activities or motivators) for their sedentary behavior and reasons for breaking up their sedentary behavior using the data collected on the previous day as a memory aid.

Although a one day measurement might be too short to give detailed information regarding patterns of sedentary behavior on different days in the week, for the purpose of this study a one-day measurement is sufficient, i.e. developing an accurate representation of 24 hours of a participants' day, to serve as a memory aid and to probe differences between perceived (believed) and actual reasons to be sedentary or to break sitting time. Moreover, a longer measurement period would be too 
"Why Older Adults Spend Time Sedentary and Break Their Sedentary Behavior: A Mixed Methods Approach Using LifeLogging Equipment" by Manon Dontje et al.

Journal of Aging and Physical Activity

(C) 2017 Human Kinetics, Inc.

Sedentary behavior in older adults

much of a burden for the participants and previous day recalls tend to be more accurate compared to longer recall periods (Diener \& Tay, 2014).

\section{Interviews}

The semi-structured interview was divided into two parts. During the first part of the interview participants were asked about what they believed were reasons (activities or motivators) for their sedentary behavior and reasons for breaking up their sedentary behavior. It should be noted that at this stage the participants did not have any knowledge about the results of in the objective measurements of their sedentary behavior. In the second part of the interview, the actual pattern of sedentary behavior was presented to the participants in the form of a personal storyboard including pictures and some basic statistics, derived from the day 1 measurements. The storyboard was used as a facilitator and memory aid, and participants were asked about their individual interpretation of the objective data. They were asked to identify and explain the reasons for being sedentary, and the reasons for breaking the sedentary bout for each period ( $>2$ minutes) and each break in sedentary behavior. Although this is still a subjective interpretation of objective data, in this paper these reasons are defined as the actual reasons in contrast to the believed reasons.

\section{Measurements}

Pattern of sedentary behavior. Participants were asked to wear an activPAL activity monitor for 1 day during waking hours (between 08.00-22.00), to measure their sedentary behavior. The activPAL is a small tri-axial accelerometer that is attached to the anterior thigh. It categorizes the subjects' activities into sitting or lying down (=sedentary behavior), standing, or walking based upon the angle of inclination of the thigh. The activPAL is a reliable and valid measure when compared to observation in measuring posture and movement and is often used as gold standard to measure sedentary behavior (Grant, Ryan, Tigbe, \& Granat, 2006; Kozey-Keadle, Libertine, Lyden, Staudenmayer, \& Freedson, 2011). This enabled the accurate detection of each period of sedentary 
"Why Older Adults Spend Time Sedentary and Break Their Sedentary Behavior: A Mixed Methods Approach Using LifeLogging Equipment" by Manon Dontje et al.

Journal of Aging and Physical Activity

(C) 2017 Human Kinetics, Inc.

Sedentary behavior in older adults

behavior for each individual on the day they were measured and the development of an accurate storyboard for that day.

Life logging. Participants were also asked to wear, on a lanyard around the neck, a

ViconRevue time-lapse camera simultaneous with the activPAL. It automatically takes photos every 50 seconds or every 10-15 seconds when in-camera sensors are activated by changes in movement, light or temperature (Kerr et al., 2013). Research has shown that the ViconRevue is a useful, objective measure in free-living conditions for identifying behaviors, contexts and environments (Kerr et al., 2013), particularly in association with other objective measures such as the accelerometer (Doherty et al., 2013; Leask et al., 2015). The ViconRevue camera and the activPAL were both synchronized using the computer clock.

Personal storyboard. For each participant, the activPAL data and camera pictures were combined into a digital narrative of the day. The starting time and duration of sedentary periods that took at least 2 minutes were identified using the activPAL data. This was used to isolate the pictures corresponding to each period of sedentary behavior ( $>2$ minutes) and to each break. The manually selected pictures were pooled together in chronological order into a personal storyboard representing sedentary periods and breaks from sedentary periods. In addition, simple descriptive statistics of the participants' sedentary behavior were computed, including the amount of time they spent sitting and their sitting behavior per hour along a time line. A schematic example of a storyboard is presented in Figure 1.

\section{Data analysis}

ActivPAL data were downloaded with activPAL software (version 7.2.32, PAL Technologies Ltd, UK). The total percentage of the day spent sedentary was calculated and descriptive statistics were used to describe individual sedentary bouts (consecutive periods) and breaks.

The interviews were recorded via a microphone and voice recording software. The interview recordings were transcribed verbatim. The interviews transcriptions were analyzed using inductive 
"Why Older Adults Spend Time Sedentary and Break Their Sedentary Behavior: A Mixed Methods Approach Using LifeLogging Equipment" by Manon Dontje et al.

Journal of Aging and Physical Activity

(c) 2017 Human Kinetics, Inc.

Sedentary behavior in older adults

thematic analysis (Braun \& Clarke, 2006). Interview quotes were grouped and sorted into different categories of reasons to remain sedentary and reasons to break sedentary time. The categories, created in an inductive way, were checked by two researchers and the opinion of a third researcher was sought in case of disagreement between the first two. Simple summary statistics representing the number of participants who reported a category were computed to provide an overview of the frequency of the categories in this study sample. Both parts of the interview were analyzed separately to obtain information about the believed reasons for (breaking) sedentary behavior as recollected by the older adults and to obtain the actual reasons as based on the individual's interpretations of what they were doing throughout their objectively measured day.

\section{Results}

\section{Study sample}

In total, 30 participants participated in this study. The mean $( \pm \mathrm{SD})$ age was $74.0( \pm 5.3)$ years and most participants were female (73\%). All participants were of Scottish origin, currently residing in Glasgow, Scotland and living independently in a flat or house. Most participants were married $(43 \%)$ or widowed (33\%). Four participants had no activPAL data due to malfunction $(n=3)$ or loss of the device $(n=1)$, and eight participants had no $(n=3)$ or partial $(n=5)$ ViconRevue data due to malfunction. One interview was not recorded due to faulty equipment. No participants lacked more than one aspect of the data.

\section{Sedentary behavior}

Participants spent on average $59.2 \%$ (range $23.1 \%$ to $93.1 \%$ ) of their monitored waking hours sedentary. One bout (= a consecutive period) of sedentary behavior was on average (mean) 19.5 minutes long (range $10.0 \mathrm{sec}$ to $166.5 \mathrm{~min}$ ). Participants accumulated on average 32 bouts (range 14 to 56) of sedentary behavior, of which on average 21 (range 11 to 35) were 2 minutes or longer. The 
"Why Older Adults Spend Time Sedentary and Break Their Sedentary Behavior: A Mixed Methods Approach Using LifeLogging Equipment" by Manon Dontje et al.

Journal of Aging and Physical Activity

(c) 2017 Human Kinetics, Inc.

Sedentary behavior in older adults

personal storyboard was only focused on bouts of at least 2 minutes. The average number of breaks in sedentary behavior was also 32 (range 14 to 56) and breaks ranged from 10.0 seconds to 139.5 minutes (mean 16.7 minutes).

\section{Believed reasons to remain sedentary}

When asked what they believed were reasons for them to remain sedentary, participants mentioned many different reasons (Figure 2). Several participants mentioned fixed TV programs they view on a daily bases as a reason for sedentary behaviour.

\footnotetext{
"Yes I watch the TV, always between say between four and six, cus I like the quiz programs and that you know". (P501)
}

Fatigue was also often mentioned as a reason to remain sedentary. One participant mentioned their fatigue was limiting them in such a way that they made an appointment for a sleep apnea clinic.

"I could fall asleep in that chair or go onto that seat and fall asleep. Fall asleep for two hours and waken up and do something and go to bed at 11 o'clock and not wake up till the next morning." (P405)

Health status was another key motivator to remain sedentary: "I have polymyalgia, it's a muscle pain. But this is what happens when you get old you know what I mean."(P407)

The most frequent reasons that people believed that kept them sedentary were: television/radio ( $48.3 \%$ of participants), fatigue (34.5\% of participants), and health status (31.0\% of participants).

\section{Actual reasons to remain sedentary}

When participants looked at their own storyboard and were asked to explain their reasons to remain seated in each sedentary period, most often reasons mentioned were eating/drinking, watching 
"Why Older Adults Spend Time Sedentary and Break Their Sedentary Behavior: A Mixed Methods Approach Using LifeLogging Equipment" by Manon Dontje et al.

Journal of Aging and Physical Activity

(C) 2017 Human Kinetics, Inc.

Sedentary behavior in older adults

television or listening to the radio, and reading or doing crossword puzzles (Figure 2). These

categories were mentioned by respectively $96.6 \%, 89.7 \%$ and $75.9 \%$ of the participants.

"I sit a lot to read. Yes I had made a coffee and read the paper with the radio on." (P502)

\section{Believed reasons to break sedentary behavior}

The reasons that the participants believed that made them break sedentary behavior could be organized into 22 different categories (Figure 3). The top three categories that were mentioned as reasons to break sedentary behavior were: domestic chores ("I do find myself moving around quite a bit for chores round the house." (P303)), walking ("I go to the city as much as I can.. If I'm not doing anything else..I must get out... I'll go on a walk and get on a bus to the city... I just don't like sitting in here." (P402))., and socializing, such as going for coffee, going to social clubs, or spending time with the grandchildren ("Yup. I had my twin grandsons up so I may have been a bit more active." (P201)). These categories were cited by $55.2 \%, 37.9 \%$ and $20.7 \%$ of participants respectively.

\section{Actual reasons to break sedentary behavior}

When participants looked at their own storyboard and were asked to explain their reasons for each break in sedentary behavior, they mentioned most often: domestic chores, food/tea preparation (Most of the time we sit down, but know most of the time when she gets up to make tea I get up to help her because she has a shaky hand and sometimes I have to carry things in for her.' (P405).), and simple tasks like getting the mail, switching lights on/off, and gathering items (Figure 3). These categories were mentioned by $86.2 \%, 82.8 \%$, and $75.9 \%$ of participants respectively when explaining the breaks in their sedentary behavior.

\section{Discussion}

Using a mixed methods approach, this study showed that there is a difference in what older adults believe or recollect to be reasons to remain sedentary or to break their sedentary time and what 
"Why Older Adults Spend Time Sedentary and Break Their Sedentary Behavior: A Mixed Methods Approach Using LifeLogging Equipment" by Manon Dontje et al.

Journal of Aging and Physical Activity

(C) 2017 Human Kinetics, Inc.

Sedentary behavior in older adults

their actual reasons are once shown their storyboard. Our findings suggest that older adults can effectively recollect reasons that they cannot influence, for example their health status, fatigue, and the weather. In contrast, they had difficulty recollecting reasons that were related to routine activities to sit down (e.g. eating and drinking) or to break sedentary behavior (e.g. food/tea preparation, selfcare and other simple daily tasks).

Watching television or listening to the radio, fatigue, and health status were most often mentioned by the older adults in this study as what they believed were reasons for them to sit down. This is in line with the findings of the other two qualitative studies (Chastin et al., 2014; GreenwoodHickman et al., 2015), but the present study showed that their believed reasons are different from their actual reasons. When the participants were presented with their personal storyboard, eating and drinking, watching television or listening to the radio, and reading or doing crossword puzzles were most often mentioned as reasons for them to sit down. An interesting finding was that whilst eating and drinking was for almost everyone a reason to sit down, only a minority had thought about this before seeing their personal story board. It is a social norm that eating and drinking should be done while sitting and according to another study, the time spent eating and drinking increases with age (Cheng, Olsen, Southerton, \& Warde, 2007). A possible explanation why most people fail to mention this as a reason to sit down is that it is such an integral part of the daily routine that they might take it for granted and not even think about it.

The same might be true for certain preferred sedentary activities, such as watching television and listening to the radio. Almost half of the sample correctly believed that watching television or listening to the radio were reasons for them to sit down. However, up to $90 \%$ of the older adults mentioned this as an explanation of a sedentary period as presented in their personal story board, meaning that about $40 \%$ of the sample had not thought about this previously. Whether social desirability played a role in the initial underreporting of watching television needs further investigation. Other studies showed that older adults spent a lot of time watching television (Bowman, 2006; Harvey, Chastin, \& Skelton, 2013). Spending a lot of time watching TV has detrimental health 
"Why Older Adults Spend Time Sedentary and Break Their Sedentary Behavior: A Mixed Methods Approach Using LifeLogging Equipment" by Manon Dontje et al.

Journal of Aging and Physical Activity

(c) 2017 Human Kinetics, Inc.

Sedentary behavior in older adults

effects, such as an increased risk of obesity, diabetes, cardio-metabolic risk factors, and mortality, possibly due to associated behaviors as snacking ( $\mathrm{Hu}, \mathrm{Li}$, Colditz, Willett, \& Manson, 2003;

Matthews et al., 2012; Thorp et al., 2010). Therefore, reducing TV time is an important target for interventions to reduce sedentary behavior, but our results suggest that it should be taken into account that many people might not be aware of, or do not want to acknowledge, how often or how long they actually sit down to watch TV. For those people who enjoy watching TV it could be a promising strategy to use the advertisements breaks for standing up or doing some light-intensity activities to make TV viewing more active.

A majority (75\%) of the older adults in this sample reported reading and doing crossword puzzles as a reason for sitting down after seeing their personal storyboard. Again, only a minority had thought about this previously as a reason to sit down. These activities are cognitively demanding, require diligent attention and are difficult to perform while moving, therefore are performed typically in a seated position. Although too much sitting should be avoided, cognitive demanding tasks like reading and crosswords are important to prevent cognitive decline in older adults and should therefore not be discredited (Brodziak, Wolinska, Kotat, \& Rozyk-Myrta, 2014; Hughes, Chang, Bilt, \& Ganguli, 2010).

Health status and fatigue were key reasons to remain sedentary, and they can act as reasons to break sedentary behavior as well. For example, some people need to keep active and moving to reduce pain and stiffness in their joints and muscles which they get from sitting too long, whereas other people need to sit down more often to prevent or manage pain in their legs or back which they get from standing or walking too long. Another study showed that inactivity is often used as a strategy to relieve pain in older adults as well,(Brown, Kirkpatrick, Swanson, \& McKenzie, 2011) but because inactivity has other detrimental health risks it is essential to treat and manage pain effectively in order to reduce sedentary behavior in older adults.

Bad weather, such as rain and darkness, was found to promote sedentary behavior by not permitting a safe or enjoyable environment for outdoor activities and by decreasing the amount of 
"Why Older Adults Spend Time Sedentary and Break Their Sedentary Behavior: A Mixed Methods Approach Using LifeLogging Equipment" by Manon Dontje et al.

Journal of Aging and Physical Activity

(c) 2017 Human Kinetics, Inc.

Sedentary behavior in older adults

reasons to break sedentary behavior, such as gardening and walking. On the other hand, good weather can be both a reason to decrease and increase sedentary behavior. For some people, good weather is a reason to sit outside to enjoy the weather, whereas for other people good weather motivates them to go outside walking or gardening. People's recollection of health status and weather as reasons to remain sedentary or to break sedentary behavior appear to be reasonably accurate, which implies that people are good at recollecting reasons that they cannot change. In addition, the examples described above show that some factors can be considered as a reason to remain sedentary for one person, but at the same time as a reason to break sedentary behavior for another person. This stresses the importance of individual recommendations and personalized interventions to reduce sedentary behavior and to increase the number of breaks in sedentary behavior.

To develop successful interventions to increase the number of breaks in sedentary behavior, it is important to know what people perceive as natural reasons to break up sedentary behavior. These could be used as starting points of interventions to increase the number of breaks without interfering with daily routine. This study showed that people most often mention domestic chores, walking, and socializing as factors that they believed were reasons for them to break sedentary behavior. This is a little different from their actual reasons, i.e. domestic chores, food or tea preparation, and performing simple tasks like switching the lights on/off and getting the mail. Although more than half of the older adults in this sample acknowledged domestic chores as a possible reason to break up periods of sitting, another approximately $30 \%$ had not thought about it without being reminded of their day with their personal storyboard. This implies that domestic chores are very much integrated into daily life, and that some people forget to think about these as reasons to break up periods of sitting. Just like preparing food or tea and doing simple tasks, domestic chores are fairly basic activities of daily living and representative of daily routines, and are not activities that illustrate conscious actions to reduce sitting. This could be harnessed by raising awareness that these daily tasks actually contribute positively to their health. 
"Why Older Adults Spend Time Sedentary and Break Their Sedentary Behavior: A Mixed Methods Approach Using LifeLogging Equipment" by Manon Dontje et al.

Journal of Aging and Physical Activity

(C) 2017 Human Kinetics, Inc.

Sedentary behavior in older adults

Since awareness is one of the prerequisites of behavioral change (Prochaska \& Norcross, 2001; Weinstein, 1988), it is important to raise awareness of the risks of prolonged sedentary behavior, and to increase awareness of their own behavior. Previous research showed that older adults underestimate the time they spent sitting (Harvey et al., 2015) and our study showed that without being reminded of their day with a personal storyboard they were not completely aware of their own reasons for prolonged sitting or reasons that made them break up their sitting. Many older adults showed difficulties in defining their reasons to remain sedentary and to break sedentary behavior, probably because sitting is very habitual and requires little conscious attention. If people are more aware about their own behavior and the risks, small adjustments can be made to change sedentary behavior and to improve health and wellbeing. Interventions should be aimed at the actual reasons that people sit, using the most frequent actual reasons that people break up their sitting such as domestic chores and food/tea preparation as a way to decrease the sedentary time and increase the number of breaks. Since results of this study show that there is a discrepancy between believed and actual reasons, people should first be made aware of their actual reasons, before an intervention can be tailor-made to the individual. This study showed that a mixed methods approach utilizing lifelogging equipment is a good strategy for this and this could potentially be used as an intervention tool.

When interpreting the results of this study, a number of things need to be taken into account. The sample size was small, the study sample was a convenience sample that consisted of mostly women, and none of the participants were using mobility aids (limitation of study heterogeneity), therefore it may not be representative to the general population. This limits the generalisibility of the findings. Sedentary behavior was objectively measured for only one day, but there can be withinsubject variability in sedentary behavior. Donaldson et al. (2016) showed that the time spent sitting per day is very stable in a 7 day period, but there may be small differences especially on weekend days (Donaldson, Montoye, Tuttle, \& Kaminsky, 2016). Weekdays can be different from weekend days, and it is possible that other determinants would have played a role in sedentary behavior when the objective measurement was done on a different day. However, although sedentary behavior and 
"Why Older Adults Spend Time Sedentary and Break Their Sedentary Behavior: A Mixed Methods Approach Using LifeLogging Equipment" by Manon Dontje et al.

Journal of Aging and Physical Activity

(C) 2017 Human Kinetics, Inc.

Sedentary behavior in older adults

the exact (prevalences of the) determinants can vary between days, this study clearly shows that there is a discrepancy between what people believe that motivates them and what actually motivates them. It needs to be taken into account that faulty equipment in a few participants may have had an impact on the quality of the personal storyboard, even though no participant lacked more than one component of data collection. Furthermore, data collection was in the Spring, but sedentary behavior and its motivators and barriers may be seasonally related. For example, rain and darkness were mentioned as barriers to decrease sedentary behavior, but these factors might be less important in Summer time.

Although more research is necessary to fully understand the complexity of sedentary behavior, it can be concluded that there is a difference in what older adults believe that are reasons for them to remain sedentary or break their sedentary time and what their actual reasons are. A mixed methods approach overcomes some of the limitations of solely qualitative research methods. Combining objective data of an activity monitor with contextual information from time lapse photos and subjective information from people regarding their own behavior, provides more indepth information about (breaking) sedentary behavior in older adults. Interventions should target the actual reasons for sedentary behavior utilizing the actual reasons for breaking sedentary behavior. A personal storyboard can be a potential tool to raise awareness about the behavior and about the discrepancy between believed and actual reasons for this behavior, which will enable finding individual and tailored strategies to reduce sedentary behavior and to increase the number of breaks in sedentary behavior. Because those strategies are tailored to the individual, they will be easier to integrate into daily life. 
"Why Older Adults Spend Time Sedentary and Break Their Sedentary Behavior: A Mixed Methods Approach Using LifeLogging Equipment" by Manon Dontje et al.

Journal of Aging and Physical Activity

(C) 2017 Human Kinetics, Inc.

Sedentary behavior in older adults

\section{Acknowledgements}

We would like to thank Alexa Starratt, Kulraj Shergill, Heather Braid, and Julia McDonald for their help with data collection and data processing.

\section{Conflict of interest statement}

The authors declare that there are no conflicts of interest. 
"Why Older Adults Spend Time Sedentary and Break Their Sedentary Behavior: A Mixed Methods Approach Using LifeLogging Equipment" by Manon Dontje et al.

Journal of Aging and Physical Activity

(C) 2017 Human Kinetics, Inc.

Sedentary behavior in older adults

\section{Figure captions:}

Figure 1 Example of a personal storyboard.

Figure 2 Reasons to remain sedentary. $*=\%$ of participants who stated this category.

Figure 3 Reasons to break up sedentary behavior. ${ }^{*}=\%$ of participants who stated this category. 
"Why Older Adults Spend Time Sedentary and Break Their Sedentary Behavior: A Mixed Methods Approach Using LifeLogging Equipment" by Manon Dontje et al.

Journal of Aging and Physical Activity

(C) 2017 Human Kinetics, Inc.

Sedentary behavior in older adults

\section{References}

Australian Government, Department of Health. (2014). Australia's physical activity and sedentary behaviour guidelines. adults. (). Common Wealth of Australia:

Berry, E., Kapur, N., Williams, L., Hodges, S., Watson, P., Smyth, G., . . Wood, K. (2007). The use of a wearable camera, SenseCam, as a pictorial diary to improve autobiographical memory in a patient with limbic encephalitis: A preliminary report. Neuropsychological Rehabilitation, 17(45), 582-601. doi:781139313

Bowman, S. A. (2006). Television-viewing characteristics of adults: Correlations to eating practices and overweight and health status. Preventing Chronic Disease, 3(2), A38-A38.

Braun, V., \& Clarke, V. (2006). Using thematic analysis in psychology. Qualitative Research in Psychology, 3(2), 77-101. doi:10.1191/1478088706qp063oa

Brodziak, A., Wolinska, A., Kotat, E., \& Rozyk-Myrta, A. (2014). Guidelines for prevention and treatment of cognitive impairment in the elderly. Medical Science Monitor : International Medical Journal of Experimental and Clinical Research, 21, 585-597.

doi:10.12659/MSM.892542

Brooke, P., \& Bullock, R. (1999). Validation of a 6 item cognitive impairment test with a view to primary care usage. International Journal of Geriatric Psychiatry, 14(11), 936-940. doi:10.1002/(SICI)1099-1166(199911)14:11<936::AID-GPS39>3.0.CO;2-1

Brown, S. T., Kirkpatrick, M. K., Swanson, M. S., \& McKenzie, I. L. (2011). Pain experience of the elderly. Pain Management Nursing : Official Journal of the American Society of Pain Management Nurses, 12(4), 190-196. doi:10.1016/j.pmn.2010.05.004 
"Why Older Adults Spend Time Sedentary and Break Their Sedentary Behavior: A Mixed Methods Approach Using LifeLogging Equipment" by Manon Dontje et al.

Journal of Aging and Physical Activity

(C) 2017 Human Kinetics, Inc.

Sedentary behavior in older adults

Browne, G., Berry, E., Kapur, N., Hodges, S., Smyth, G., Watson, P., \& Wood, K. (2011). SenseCam improves memory for recent events and quality of life in a patient with memory retrieval difficulties. Memory, 19(7), 713-722.

Chastin, S. F. M., Fitzpatrick, N., Andrews, M., \& DiCroce, N. (2014). Determinants of sedentary behavior, motivation, barriers and strategies to reduce sitting time in older women: A qualitative investigation. International Journal of Environmental Research and Public Health, 11(1), 773791. doi:10.3390/ijerph110100773

Chau, J. Y., Grunseit, A. C., Chey, T., Stamatakis, E., Brown, W. J., Matthews, C. E., . . van der Ploeg, H. P. (2013). Daily sitting time and all-cause mortality: A meta-analysis. PLoS ONE, 8(11) doi:10.1371/journal.pone.0080000

Cheng, S., Olsen, W., Southerton, D., \& Warde, A. (2007). The changing practice of eating: Evidence from UK time diaries, 1975 and 20001. The British Journal of Sociology, 58(1), 39-61. doi:10.1111/j.1468-4446.2007.00138.x

Davis, M. G., Fox, K. R., Hillsdon, M., Sharp, D. J., Coulson, J. C., \& Thompson, J. L. (2011). Objectively measured physical activity in a diverse sample of older urban UK adults. Medicine and Science in Sports and Exercise, 43(4), 647-654. doi:10.1249/MSS.0b013e3181f36196

de Rezende, L. F. M., Rey-López, J. P., Matsudo, V. K. R., \& Luiz, O. d. C. (2014). Sedentary behavior and health outcomes among older adults: A systematic review. BMC Public Health, 14(1), 333. doi:10.1186/1471-2458-14-333

Department of Health. (2011). UK physical activity guidelines for older adults (fact sheet 5). (). London, England: Deparment of Health. 
"Why Older Adults Spend Time Sedentary and Break Their Sedentary Behavior: A Mixed Methods Approach Using LifeLogging Equipment" by Manon Dontje et al.

Journal of Aging and Physical Activity

(C) 2017 Human Kinetics, Inc.

Sedentary behavior in older adults

Department of Health, Physical Activity, Health Improvement and Protection. (2011). Start active, stay active: A report on physical activity from the four home countries' chief medical officers. (). London, United Kingdom:

Diener, E., \& Tay, L. (2014). Review of the day reconstruction method (DRM). Social Indicators Research, 116(1), 255-267. doi:10.1007/s11205-013-0279-х

Dogra, S., \& Stathokostas, L. (2012). Sedentary behavior and physical activity are independent predictors of successful aging in middle-aged and older adults. Journal of Aging Research, 2012, 10.1155/2012/190654. doi:190654

Doherty, A. R., Kelly, P., Kerr, J., Marshall, S., Oliver, M., Badland, H., . . Foster, C. (2013). Using wearable cameras to categorise type and context of accelerometer-identified episodes of physical activity. International Journal of Behavioral Nutrition and Physical Activity, 10, 22. doi:10.1186/1479-5868-10-22

Donaldson, S. C., Montoye, A. H., Tuttle, M. S., \& Kaminsky, L. A. (2016). Variability of objectively measured sedentary behavior. Medicine and Science in Sports and Exercise, 48(4), 755-761. doi:10.1249/MSS.0000000000000828 [doi]

Erzberger, C., \& Prein, G. (1997). Triangulation: Validity and empirically-based hypothesis construction. Quality and Quantity, 31(2), 141-154. doi:10.1023/A:1004249313062

Grant, P. M., Ryan, C. G., Tigbe, W. W., \& Granat, M. H. (2006). The validation of a novel activity monitor in the measurement of posture and motion during everyday activities. British Journal of Sports Medicine, 40(12), 992-997. doi:10.1136/bjsm.2006.030262

Greenwood-Hickman, M. A., Renz, A., \& Rosenberg, D. E. (2015). Motivators and barriers to reducing sedentary behavior among overweight and obese older adults. The Gerontologist, doi:10.1093/geront/gnu163 
"Why Older Adults Spend Time Sedentary and Break Their Sedentary Behavior: A Mixed Methods Approach Using LifeLogging Equipment" by Manon Dontje et al.

Journal of Aging and Physical Activity

(C) 2017 Human Kinetics, Inc.

Sedentary behavior in older adults

Harvey, J. A., Chastin, S. F., \& Skelton, D. A. (2015). How sedentary are older people? A systematic review of the amount of sedentary behavior. Journal of Aging and Physical Activity, 23(3), 471487. doi:10.1123/japa.2014-0164

Harvey, J. A., Chastin, S. F. M., \& Skelton, D. A. (2013). Prevalence of sedentary behavior in older adults: A systematic review. International Journal of Environmental Research and Public Health, 10(12), 6645-6661. doi:10.3390/ijerph10126645

Hu, F. B., Li, T. Y., Colditz, G. A., Willett, W. C., \& Manson, J. E. (2003). Television watching and other sedentary behaviors in relation to risk of obesity and type 2 diabetes mellitus in women. Jama-Journal of the American Medical Association, 289(14), 1785-1791. doi:10.1001/jama.289.14.1785

Hughes, T., Chang, C. H., Bilt, J. V., \& Ganguli, M. (2010). Engagement in reading and hobbies and risk of incident dementia: The MoVIES project. American Journal of Alzheimer's Disease and Other Dementias, 25(5), 432-438. doi:10.1177/1533317510368399

Kelly, P., Marshall, S. J., Badland, H., Kerr, J., Oliver, M., Doherty, A. R., \& Foster, C. (2013). An ethical framework for automated, wearable cameras in health behavior research. American Journal of Preventive Medicine, 44(3), 314-319. doi:10.1016/j.amepre.2012.11.006 [doi]

Kelly, P., Thomas, E., Doherty, A., Harms, T., Burke, O., Gershuny, J., \& Foster, C. (2015). Developing a method to test the validity of 24 hour time use diaries using wearable cameras: A feasibility pilot. PloS One, 10(12), e0142198. doi:10.1371/journal.pone.0142198 [doi]

Kerr, J., Marshall, S. J., Godbole, S., Chen, J., Legge, A., Doherty, A. R., . . Foster, C. (2013). Using the SenseCam to improve classifications of sedentary behavior in free-living settings. American Journal of Preventive Medicine, 44(3), 290-296. doi:10.1016/j.amepre.2012.11.004 
"Why Older Adults Spend Time Sedentary and Break Their Sedentary Behavior: A Mixed Methods Approach Using LifeLogging Equipment" by Manon Dontje et al.

Journal of Aging and Physical Activity

(C) 2017 Human Kinetics, Inc.

Sedentary behavior in older adults

Kozey-Keadle, S., Libertine, A., Lyden, K., Staudenmayer, J., \& Freedson, P. S. (2011). Validation of wearable monitors for assessing sedentary behavior. Medicine and Science in Sports and Exercise, 43(8), 1561-1567. doi:10.1249/MSS.0b013e31820ce174

Leask, C. F., Harvey, J. A., Skelton, D. A., \& Chastin, S. F. M. (2015). Exploring the context of sedentary behaviour in older adults (what, where, why, when and with whom). European Review of Aging and Physical Activity, In press

Matthews, C. E., Chen, K. Y., Freedson, P. S., Buchowski, M. S., Beech, B. M., Pate, R. R., \& Troiano, R. P. (2008). Amount of time spent in sedentary behaviors in the united states, 20032004. American Journal of Epidemiology, 167(7), 875-881. doi:10.1093/aje/kwm390

Matthews, C. E., George, S. M., Moore, S. C., Bowles, H. R., Blair, A., Park, Y., . . Schatzkin, A. (2012). Amount of time spent in sedentary behaviors and cause-specific mortality in US adults123. The American Journal of Clinical Nutrition, 95(2), 437-445. doi:019620

Nicolai, S., Benzinger, P., Skelton, D. A., Aminian, K., Becker, C., \& Lindemann, U. (2010). Day-today variability of physical activity of older adults living in the community. Journal of Aging and Physical Activity, 18(1), 75-86.

Prochaska, J., \& Norcross, J. (2001). Stages of change. Psychotherapy, 38(4), 443-448. doi:10.1037/0033-3204.38.4.443

Rezende, L. F. M., Rey-Lopez, J. P., Matsudo, V. K. R., \& Luiz Odo, C. (2014). Sedentary behavior and health outcomes among older adults: A systematic review. BMC Public Health, 14, 3332458-14-333. doi:1471-2458-14-333

Sedentary Behaviour Research, N. (2012). Letter to the editor: Standardized use of the terms "sedentary" and "sedentary behaviours". Applied Physiology, Nutrition, and Metabolism = Physiologie Appliquee, Nutrition Et Metabolisme, 37(3), 540-542. doi:10.1139/h2012-024 [doi] 
"Why Older Adults Spend Time Sedentary and Break Their Sedentary Behavior: A Mixed Methods Approach Using LifeLogging Equipment" by Manon Dontje et al.

Journal of Aging and Physical Activity

(C) 2017 Human Kinetics, Inc.

Sedentary behavior in older adults

Smith, R. (1994). Validation and reliability of the elderly mobility scale. Physiotherapy, 80(11), 744747.

Thorp, A. A., Healy, G. N., Owen, N., Salmon, J., Ball, K., Shaw, J. E., . . Dunstan, D. W. (2010). Deleterious associations of sitting time and television viewing time with cardiometabolic risk biomarkers: Australian diabetes, obesity and lifestyle (AusDiab) study 2004-2005. Diabetes Care, 33(2), 327-334. doi:0493

van Uffelen, J.,G.Z., Heesch, K. C., Hill, R. L., \& Brown, W. J. (2011). A qualitative study of older adults' responses to sitting-time questions: Do we get the information we want? BMC Public Health, 11, 458-458. doi:10.1186/1471-2458-11-458

Weinstein, N. D. (1988). The precaution adoption process. Health Psychology : Official Journal of the Division of Health Psychology, American Psychological Association, 7(4), 355-386. 